QUANTUM PROBABILITY

BANACH CENTER PUBLICATIONS, VOLUME 73

INSTITUTE OF MATHEMATICS

POLISH ACADEMY OF SCIENCES

WARSZAWA 2006

\title{
QUANTUM RANDOM WALK REVISITED
}

\author{
KALYAN B. SINHA \\ Indian Statistical Institute \\ 7, SJS Sansanwal Marg \\ New Delhi 110016, India \\ E-mail:kbs@isid.ac.in
}

\begin{abstract}
In the framework of the symmetric Fock space over $L^{2}\left(\mathbb{R}_{+}\right)$, the details of the approximation of the four fundamental quantum stochastic increments by the four appropriate spin-matrices are studied. Then this result is used to prove the strong convergence of a quantum random walk as a map from an initial algebra $\mathcal{A}$ into $\mathcal{A} \otimes \mathcal{B}\left(\right.$ Fock $\left.\left(L^{2}\left(\mathbb{R}_{+}\right)\right)\right)$to a ${ }^{*}$-homomorphic quantum stochastic flow.
\end{abstract}

1. Introduction. As a sequel to the early works of Parthasarathy [Par 1987] and of Lindsay and Parthasarathy [L-P 1988], Attal and Pautrat [A-P 2006] revived the idea of quantum random walk and showed the weak convergence of a sequence of unitaries to the unitary process solution of a Hudson-Parthasarathy equation with constant bounded operator coefficients in an initial Hilbert space. However, unlike in [Par 1987], they work in one Hilbert space, viz. the symmetric (Bosonic) Fock space, with the 'walks' taking place in an infinite dimensional 'toy Fock space' which is a subspace of the Fock space. However, in both cases the authors only prove weak convergence. They therefore cannot conclude unitarity of the limiting operator process and thus do not have the homomorphic property for the resulting mapping process. For the motivation from classical Markov chains set in algebraic language, we refer the reader to the work of Lindsay and Parthasarathy [L-P 1988]; we also use mostly their notations and work only in the Heisenberg picture, i.e. with the processes as maps on the algebra of the observables of the system [A-F-L 1982].

Here we consider only the case with bounded coefficients of the stochastic process coming in a natural way from the one-step quantum random walk. However, we have

2000 Mathematics Subject Classification: 81S25, 46N50, 60H10.

The author acknowledges gratefully the discussions with Professor S. Attal during a visit of the author to the Institute Joseph Fourier, University of Grenoble, France as a part of the research project 2301-1 under the auspices of Indo-French Centre for the promotion of Advanced Research.

The paper is in final form and no version of it will be published elsewhere. 
found it useful to separate the two issues in the study of quantum stochastic diffusion processes viz. the one on the existence of a solution of a quantum stochastic differential equation (q.s.d.e.) and other on *-homomorphic property of the solution as a map on the algebra of observables. In fact, we assume the existence of a strongly continuous solution of the q.s.d.e. for the diffusion, and also assume the existence of a one-step quantum walk given by *homomorphisms depending on the step-size which in a suitable sense approximates the coefficients driving the diffusion as the step-size converges to zero. Here we work with bounded coefficients so that the assumption on existence of solutions holds, by standard quantum stochastic theory.

The motivation behind such a separation of assumptions is that in the case of unbounded coefficients one may be able to find a solution of the q.s.d.e. by some other methods (see e.g. [A-K 2001] and [L-W 2004]). Then the hope is that under suitable conditions the method indicated here may be modified to show strong convergence of the associated quantum random walk, thereby proving the *-homomorphic property of the solution.

2. Notations and preliminaries. All tensor products between von Neumann algebras are meant in the ultraweak sense. We begin by fixing a basis $\left\{N_{k}\right\}_{k=1}^{4}$ of $\mathcal{B}\left(\mathbb{C}^{2}\right)$ so that $\sum_{j=1}^{4} b_{j} N_{j}=I$ for some constants $b_{1}, \ldots, b_{4}$, in particular, we choose

$$
N_{1}=\left[\begin{array}{ll}
1 & 0 \\
0 & 0
\end{array}\right], N_{2}=\left[\begin{array}{ll}
0 & 1 \\
0 & 0
\end{array}\right], N_{3}=\left[\begin{array}{ll}
0 & 0 \\
1 & 0
\end{array}\right], N_{4}=\left[\begin{array}{ll}
0 & 0 \\
0 & 1
\end{array}\right] .
$$

In such a case, $b_{2}=b_{3}=0$ and $b_{1}=b_{4}=1$ and we also note that the $N_{j}$ 's satisfy the following algebraic relations:

$$
\begin{aligned}
& N_{3}=N_{2}^{*}, N_{2}^{2}=N_{3}^{2}=0, \\
& N_{1}=N_{2} N_{3}, N_{4}=N_{3} N_{2}, \\
& N_{2} N_{3}+N_{3} N_{2}=N_{1}+N_{4}=I .
\end{aligned}
$$

We also observe that these are similar to the canonical anti-commutation relations (CAR) or equivalently the spin algebra relations satisfied by the Pauli matrices, and this is the reason why the authors of [L-P 1988] named the associated random walk as quantum spin random walk.

Let $\mathcal{A} \subseteq \mathcal{B}(\mathfrak{h})$ be a von Neumann algebra of system observables where $\mathfrak{h}$ is a separable Hilbert space and let $\alpha: \mathcal{A} \rightarrow \mathcal{A} \otimes \mathcal{B}\left(\mathcal{C}^{2}\right)$ be the basic unital *-homomorphism or the one step random walk. This means that we can write for $x \in \mathcal{A}$,

$$
\alpha(x)=\sum_{j=1}^{4} \alpha_{j}(x) \otimes N_{j}
$$

and this also means that the equalities $\alpha(x y)=\alpha(x) \alpha(y)$ and $\alpha(x)^{*}=\alpha\left(x^{*}\right)$ will imply

$$
\begin{gathered}
\alpha_{j}(x y)=\sum C_{k l}^{j} \alpha_{k}(x) \alpha_{l}(y), \quad \alpha_{j}(I)=b_{j}, \\
\alpha_{1}(x)^{*}=\alpha_{1}\left(x^{*}\right), \alpha_{2}(x)=\alpha_{3}\left(x^{*}\right), \alpha_{3}(x)=\alpha_{2}\left(x^{*}\right), \alpha_{4}(x)=\alpha_{4}\left(x^{*}\right),
\end{gathered}
$$

where $C_{k l}^{j}$ is determined by $N_{k} N_{l}=\sum_{j} C_{k l}^{j} N_{j}$ as can be seen in (1). 
We can now construct the n-step quantum spin random walk by the following recursive process:

$$
J_{0}(x)=x, \quad J_{1}(x)=\alpha(x), \quad J_{n}(x)=\sum_{j=1}^{4} J_{n-1}\left(\alpha_{j}(x) \otimes N_{j}\right) .
$$

It is clear that (as in [L-P 1988]) for each $n, J_{n}: \mathcal{A} \rightarrow \mathcal{A} \otimes \mathcal{B}\left(\mathbb{C}^{2}\right)^{\otimes n}$ is a *-homomorphism.

Next, in order to pass to the diffusion limit of a quantum stochastic process, we need to embed the structure in a Fock space. Thus consider the symmetric (Bosonic) Fock space $\mathcal{H} \equiv \Gamma\left(L^{2}\left(\mathbb{R}_{+}\right)\right)=\mathcal{H} \equiv \mathbb{C} \oplus \sum_{n=1}^{\oplus} L^{2}\left(\mathbb{R}_{+}\right){ }^{(}{ }^{n}$, where $\mathrm{S}^{n}$ denotes $n$-fold symmetric tensor product. For any $f \in L^{2}\left(\mathbb{R}_{+}\right)$, we denote by $e(f)$ the exponential vector given by

$$
e(f)=1 \oplus f \oplus \frac{f^{\otimes 2}}{\sqrt{2}} \oplus \cdots \oplus \frac{f^{\otimes n}}{\sqrt{n}} \oplus \cdots
$$

so that

$$
\langle e(f), e(g)\rangle=\exp (<f, g>) .
$$

We also note the standard Fock space properties:

(i) continuous tensor product property: $L^{2}\left(\mathbb{R}_{+}\right) \cong L^{2}(0, a) \oplus L^{2}(a, b) \oplus L^{2}(b, \infty)$ implies $\Gamma\left(L^{2}\left(\mathbb{R}_{+}\right)\right) \simeq \Gamma\left(L^{2}(0, a)\right) \otimes \Gamma\left(L^{2}(a, b)\right) \otimes \Gamma\left(L^{2}(b, \infty)\right)$ for $0 \leq a \leq b \leq \infty ;$

(ii) if we define, for $0 \leq a \leq b<\infty, \theta(a, b)$ to be the unitary isomorphism from $L^{2}[0,1]$ onto $L^{2}[a, b]$ given by $(\theta(a, b) f)(s)=(b-a)^{-1 / 2} f\left((b-a)^{-1}(s-a)\right)$, where $f \in L^{2}[0,1]$, then this lifts to a unitary isomorphism of $\mathcal{H}(0,1)$ onto $\mathcal{H}(a, b)$ by second quantization where we have written $\mathcal{H}(a, b)=\Gamma\left(L^{2}(a, b)\right)$.

We have already observed that (1) is a representation of the CAR. By the results of Hudson and Parthasarathy [H-P 1986] the CAR also admit a representation in the Fock space $\mathcal{H}$ as follows:

$$
N_{2}=\int_{0}^{1} \Gamma\left(R_{s}\right) A(d s), \quad N_{3}=\int_{0}^{1} \Gamma\left(R_{s}\right) A^{\dagger}(d s),
$$

where $A, A^{\dagger}$ and $\Lambda$ are the three fundamental (martingale) processes in $\mathcal{H}$ (see Par 1992), $\Gamma\left(R_{s}\right)$ is the second quantization of the reflection operator $R_{s}$ in $L^{2}\left(\mathbb{R}_{+}\right)$defined as

$$
\left(R_{s} f\right)(t)=\left\{\begin{array}{cl}
-f(t) & \text { if } t \leq s \\
f(t) & \text { if } t>s
\end{array}\right.
$$

and $N_{1}$ and $N_{4}$ are obtained by using the relations in (1). In the standard notation of the CAR, $N_{2}=a$ and $N_{3}=a^{+}$, moreover $N_{4}=a^{+} a$, the number operator, and the $N_{k}$ 's so represented are bounded operators in $\mathcal{H}(0,1)$.

We also set, for $0 \leq a \leq b \leq \infty$ and $l=1,2,3,4$,

$$
N_{l}(a, b)=\Gamma(\theta(a, b)) N_{l} \Gamma(\theta(a, b))^{-1}
$$

so that the $N_{l}(a, b)$ 's are bounded operators in $\mathcal{H}(a, b)$.

Next suppose that for each non-zero positive number h, we are given a basic*homomorphism $\alpha(h, \cdot): \mathcal{A} \rightarrow \mathcal{A} \otimes B(0, h)$ as in (2). Then we can proceed to construct 
the $n$-step random walk as follows: for $t \geq 0$,

$$
\begin{gathered}
J_{0}^{(h)}(x)=x, \quad J_{h}^{(h)}(x)=\sum_{k=1}^{4} \alpha_{k}(h, x) \otimes N_{k}(0, h), \\
J_{t}^{(h)}(x)=\sum_{k=1}^{4} J_{\frac{(h)}{n-1} h}\left(\alpha_{k}(h, x)\right) \otimes N_{k}(\overline{n-1} h, n h),
\end{gathered}
$$

where $\overline{n-1} h<t \leq n h$.

From the construction above, two facts follow easily:

(i) for each $x \in \mathcal{A}$ and $t \geq 0$

$J_{t}^{(h)}(x) \in \mathcal{A} \otimes \mathcal{B}(0, h) \otimes \mathcal{B}(h, 2 h) \otimes \cdots \otimes \mathcal{B}(\overline{n-1} h, n h) \subseteq \mathcal{A} \otimes \mathcal{B}(0, n h) \subseteq \mathcal{A} \otimes \mathcal{B}(\mathcal{H})$

where we have used the notation $\mathcal{B}(a, b)=\mathcal{B}(\mathcal{H}(a, b))$;

(ii) since each $J_{t}^{(h)}$ is *-homomorphic and unital, in particular it satisfies

$$
\left\|J_{t}^{(h)}(x)\right\| \leq\|x\|
$$

Here we also collect a result from pages 185-186 of [Par 1992] which will be used more than once in the sequel.

Proposition 2.1 Let $\left\{X_{j}(t)\right\}_{j=1,2}$ be stochastic processes given by

$$
X_{j}(t)=\sum_{k=1}^{n} L_{j}\left(t_{k-1}\right)\left[M_{j}\left(t_{k}\right)-M_{j}\left(t_{k-1}\right)\right]
$$

where $t_{0}<t_{1}<\cdots<t_{n}=t$ and $M_{j}$ is any martingale process (such as a linear combination of $A, A^{+}$and $\left.\Lambda\right)$. Then for $u, v \in \mathfrak{h}$ and $f, g \in L^{2}\left(\mathbb{R}_{+}\right)$,

$$
\begin{aligned}
\left\langle X_{1}(t) u e(f), X_{2}(t) v e(g)\right\rangle \\
=\sum_{j=1}^{n}\left\langle L_{1}\left(t_{j-1}\right) u e\left(f_{\left.t_{j-1}\right]}\right), L_{2}\left(t_{j-1}\right) v e\left(g_{t_{j-1}}\right)\right\rangle \\
\quad \cdot\left\langle\left(M_{1}\left(t_{j}\right)-M_{1}\left(t_{j-1}\right)\right) e\left(f_{\left[t_{j-1}\right.}\right),\left(M_{2}\left(t_{j}\right)-M_{2}\left(t_{j-1}\right)\right) e\left(g_{\left[t_{j-1}\right.}\right)\right\rangle \\
+\sum_{j=1}^{n}\left\langle X_{1}\left(t_{j-1}\right) u e\left(f_{\left.t_{j-1}\right]}\right), L_{2}\left(t_{j-1}\right) v e\left(g_{\left.t_{j-1}\right]}\right)\right\rangle\left\langle e\left(f_{\left[t_{j-1}\right.}\right),\left(M_{2}\left(t_{j}\right)-M_{2}\left(t_{j-1}\right)\right) e\left(g_{\left[t_{j-1}\right.}\right)\right\rangle \\
+ \\
\quad \sum_{j=1}^{n}\left\langle L_{1}\left(t_{j-1}\right) u e\left(f_{\left.t_{j-1}\right]}\right), X_{2}\left(t_{j-1}\right) v e\left(g_{\left.t_{j-1}\right]}\right)\right\rangle \\
\quad \cdot\left\langle\left(M_{1}\left(t_{j}\right)-M_{1}\left(t_{j-1}\right)\right) e\left(f_{\left[t_{j-1}\right.}\right), e\left(g_{\left[t_{j-1}\right.}\right)\right\rangle .
\end{aligned}
$$

3. Approximation of the fundamental processes. Here we derive some useful estimates regarding the behaviour of the $N_{k}$ 's in relation to the fundamental processes in the Fock space and for this it is convenient to use the notation:

$$
\Lambda_{1}(s)=s, \quad \Lambda_{2}(s)=A(s), \quad \Lambda_{3}(s)=A^{\dagger}(s), \quad \Lambda_{4}(s)=\Lambda(s),
$$

and

$$
\varepsilon_{1}=1, \quad \varepsilon_{2}=\varepsilon_{3}=1 / 2, \quad \varepsilon_{4}=0
$$


Lemma 3.1. Let $f, g \in \mathcal{M} \equiv B C^{1}\left(\mathbb{R}_{+}\right) \cap L^{2}\left(\mathbb{R}_{+}\right)$and $l$ be a natural number. Then for $0<h<1, k=1,2,3,4$,

(i) $\quad\left|\left\langle e\left(g_{[\overline{l-1} h, l h]}\right),\left[h^{\varepsilon_{k}} N_{k}(\overline{l-1} h, l h)-\Lambda_{k}(\overline{l-1} h, l h)\right] e\left(f_{[\overline{l-1} h, l h]}\right)\right\rangle\right| \leq C_{1} h^{2}$,

(ii) $\quad \|\left[h^{\varepsilon_{k}} N_{k}(\overline{l-1} h, l h)-\Lambda_{k}(\overline{l-1} h, l h)\right] e\left(g_{[\overline{l-1} h, l h]} \| \leq\left\{\begin{array}{cc}C_{2} h^{\frac{3}{2}} & \text { for } k=1,2, \\ C_{2} h & \text { for } k=3,4 .\end{array}\right.\right.$

where $C_{1}$ and $C_{2}$ are two positive constants depending on $g, f$ and on $g$ respectively. Here also for an interval $\Delta \subseteq \mathbb{R}_{+}, g_{\Delta}$ means $g \chi_{\Delta}$ and $B C^{1}\left(\mathbb{R}_{+}\right)$means the space of once continuously differentiable functions on $\mathcal{R}_{+}$with the function as well as its derivative bounded. In the following for the brevity of writing, we shall write $g_{l-1]}, g_{[l]}, g_{[l}$ for $g_{[0, \overline{l-1} h]}, g_{[\overline{l-1} h, l h]}$ and $g_{[l h}$ respectively and $N_{k}[l]$ and $\Lambda_{k}[l]$ for $N_{k}(\overline{l-1} h, l h)$ and $\Lambda_{k}(\overline{l-1} h, l h)$ respectively.

Proof. (i)

$$
\begin{aligned}
& \left\langle e\left(g_{[l]}\right),\left(N_{4}[l]-\Lambda[l]\right) e\left(f_{[l]}\right)\right\rangle \\
& \quad=\frac{1}{h} \int_{\overline{l-1} h}^{l h} \bar{g}(s) d s \int_{\overline{l-1} h}^{l h} f(\tau) d \tau e^{\left\langle R_{s} g_{[l]}, R_{\tau} f_{[l]}\right\rangle}-\int_{\overline{l-1} h}^{l h} \bar{g}(s) f(s) d s e^{\left\langle g_{[l]} f_{[l]}\right\rangle} .
\end{aligned}
$$

Noting that

$$
R_{s} R_{\tau} f(t)= \begin{cases}-f(t) & \text { if } t \in[s \wedge \tau, s \vee \tau] \\ f(t) & \text { otherwise, }\end{cases}
$$

we get that the L.H.S. in (i) equals

$$
\begin{array}{r}
\frac{1}{h} \int_{\overline{l-1} h}^{l h} \bar{g}(s) d s \int_{\overline{l-1} h}^{l h} f(\tau) d \tau\left[\exp \left\{\int_{\overline{l-1} h}^{s \Lambda \tau} \bar{g} f-\int_{s \Lambda \tau}^{s \vee \tau} \bar{g} f+\int_{s \vee \tau}^{l h} \bar{g} f\right\}-\exp \left\{\int_{\overline{l-1} h}^{l h} \bar{g} f\right\}\right] \\
+\exp \left\{\int_{\overline{l-1} h}^{l h} \bar{g} f\right\} \cdot\left[\frac{1}{h} \int_{\overline{l-1} h}^{l h} d s \bar{g}(s) \int_{\overline{l-1} h}^{l h} f(\tau) d \tau-\int_{\overline{l-1} h}^{l h} \bar{g}(s) f(s) d s\right] \\
\equiv \mathbf{I}_{1}+\mathbf{I}_{2} .
\end{array}
$$

We have

$$
\begin{aligned}
&\left|\mathbf{I}_{1}\right| \leq h\|g\|_{\infty}\|f\|_{\infty} \exp (\|g\|\|f\|) \sup _{s, \tau, \in[\overline{l-1} h, l h]} \mid 1- \exp \left\{-2 \int_{s \wedge \tau}^{s \vee \tau} \bar{g} f\right\} \mid \\
& \leq 2 h^{2}\|g\|_{\infty}^{2}\|f\|_{\infty}^{2} \exp (3\|g\|\|f\|),
\end{aligned}
$$

and

$$
\begin{aligned}
\left|\mathbf{I}_{2}\right| & \leq\left|\exp \left(\int_{\overline{l-1} h}^{l h} \bar{g} f\right) \| \frac{1}{h} \int_{\overline{l-1} h}^{l h} \bar{g}(s) d s \int_{\overline{l-1} h}^{l h}[f(\tau)-f(s)] d \tau\right| \\
& \leq 2 \exp (\|g\|\|f\|)\|g\|_{\infty}\left\|f^{\prime}\right\|_{\infty} h^{2},
\end{aligned}
$$

proving that

$$
\mid\left\langle e\left(g_{[l]}\right),\left(N_{4}[l]-\Lambda[l]\right) e\left(f_{[l]}\right\rangle\right| \leq C_{1} h^{2} .
$$

Next,

$$
\left.\left\langle e\left(g_{[l]}\right),\left(h N_{1}[l]-h\right) e\left(f_{[l]}\right)\right\rangle=-h\left\{\left\langle e\left(g_{[l]}\right)\left\{N_{4}[l]-\Lambda[l]\right\} e\left(f_{[l]}\right)\right\rangle+\left\langle e\left(g_{[l]}\right), \Lambda[l] e\left(f_{[l}\right]\right)\right\rangle\right\}
$$

and thus by the previous estimates, $\mid$ L.H.S. $\mid \leq C_{1}^{\prime \prime} h^{3}+C_{1}^{\prime} h^{2}$ for small $h$. 
Next,

$$
\begin{aligned}
\left\langle e\left(g_{[l]}\right),\left(h^{\frac{1}{2}} N_{3}[l]-\right.\right. & \left.\left.A^{\dagger}[l]\right) e\left(f_{[l]}\right)\right\rangle \\
& =\int_{\overline{l-1} h}^{l h} \bar{g}(s) d s\left\langle e\left(g_{[l]}\right),\left(\Gamma\left(R_{s}\right)-I\right) e\left(f_{[l]}\right)\right\rangle \\
& =\int_{\overline{l-1} h}^{l h} \bar{g}(s) d s\left\{\exp \left(-\int_{\overline{l-1} h}^{s} \bar{g} f+\int_{s}^{l h} \bar{g} f\right)-\exp \left(\int_{\overline{l-1} h}^{l h} \bar{g} f\right)\right\}
\end{aligned}
$$

and an estimate similar to the earlier one leads to

$$
\left|\left\langle e\left(g_{[l]}\right),\left(h^{\frac{1}{2}} N_{3}[l]-A^{\dagger}[l]\right) e\left(f_{[l]}\right)\right\rangle\right| \leq 2\|g\|_{\infty}^{2} \exp (3\|g\|\|f\|)\|f\|_{\infty} h^{2} .
$$

Finally,

$$
\left|\left\langle e\left(g_{[l]}\right),\left(h^{\frac{1}{2}} N_{2}[l]-A[l]\right) e\left(f_{[l]}\right)\right\rangle\right|=\left|\left\langle e\left(f_{[l]}\right),\left(h^{\frac{1}{2}} N_{3}[l]-A^{\dagger}[l]\right) e\left(g_{[l]}\right)\right\rangle\right|
$$

and a similar estimate follows from the previous one.

(ii) We have

$$
\begin{aligned}
\left\|\left(h N_{1}[l]-h\right) e\left(g_{[l]}\right)\right\|^{2} & =h^{2}\left\langle e\left(g_{[l]}\right), N_{4}[l] e\left(g_{[l]}\right)\right\rangle \\
& =h^{2}\left\{\left\langle e\left(g_{[l]}\right),\left(N_{4}[l]-\Lambda[l]\right) e\left(g_{[l]}\right)\right\rangle+\left\langle e\left(g_{[l]}\right), \Lambda[l] e\left(g_{[l]}\right)\right\rangle\right\} \\
& \leq h^{2}\left(C^{\prime} h^{2}+C^{\prime \prime} h\right) \leq C_{2}^{2} h^{3}
\end{aligned}
$$

giving the required estimate. Similarly,

$$
\left\|\left\{h^{\frac{1}{2}} N_{2}[l]-A[l]\right\} e\left(g_{[l]}\right)\right\|=\left\|\int_{\overline{l-1} h}^{l h} g(s) d s\left[e\left(R_{s} g_{[l]}\right)-e\left(g_{[l]}\right)\right]\right\| \leq \text { Const } h^{\frac{3}{2}},
$$

since

$$
\begin{aligned}
\left\|e\left(R_{s} g_{[l]}\right)-e\left(g_{[l]}\right)\right\|^{2} & =2\left[\exp \left(\int_{\overline{l-1} h}^{l h}|g|^{2}\right)-\exp \left(-\int_{\overline{l-1} h}^{s}|g|^{2}+\int_{s}^{l h}|g|^{2}\right)\right] \\
& \leq 4 \exp \left(\|g\|^{2}\right)\|g\|_{\infty}^{2} h
\end{aligned}
$$

Next,

$$
\begin{aligned}
\left\|\left(\sqrt{h} N_{3}[l]-A^{\dagger}[l]\right) e\left(g_{[l]}\right)\right\| & =\left\|\int_{\overline{l-1} h}^{l h} d A^{\dagger}(s)\left\{e\left(R_{s} g_{[l]}\right)-e\left(g_{[l]}\right)\right\}\right\| \\
& \leq C^{\prime \prime \prime} \sqrt{\int_{\overline{l-1} h}^{l h}\left(1+|g(s)|^{2}\right)\left\|e\left(R_{s} g_{[l]}\right)-e\left(g_{[l]}\right)\right\|^{2}} \leq C_{2} h,
\end{aligned}
$$

where we have used the estimate in the previous paragraph. Finally,

$$
\begin{aligned}
& \left\|\left(N_{4}[l]-\Lambda[l]\right) e\left(g_{[l]}\right)\right\|^{2} \\
& \quad=\left\langle e\left(g_{[l]}\right), N_{4}[l] e\left(g_{[l]}\right)\right\rangle+\left\langle e\left(g_{[l]}\right), \Lambda[l]^{2} e\left(g_{[l]}\right)\right\rangle-2 R e\left\langle N_{4}[l] e\left(g_{[l]}\right), \Lambda[l] e\left(g_{[l]}\right)\right\rangle .
\end{aligned}
$$

By the quantum Ito formula, we have

$$
d_{t} \Lambda[a, t]^{2}=2 \Lambda[a, t] d \Lambda(t)+d \Lambda(t)
$$

or,

$$
\Lambda[l]^{2}=\int_{\overline{l-1} h}^{l h}(2 \Lambda(\overline{l-1} h, t)+1) d \Lambda(t)
$$


Therefore,

$$
\begin{aligned}
\left\langle e\left(g_{[l]}(, \Lambda[l]]^{2} e\left(g_{[l]}\right)\right\rangle\right. & =\int_{\overline{l-1} h}^{l h}|g(s)|^{2} d s\left\langle e\left(g_{[l]}\right),\{2 \Lambda(\overline{l-1} h, s)+1\} e\left(g_{[l]}\right)\right\rangle \\
& =\int_{\overline{l-1} h}^{l h}|g(s)|^{2} d s\left\{2 \int_{\overline{l-1} h}^{s}|g(\tau)|^{2} d \tau+1\right\} \exp \left(\left\|g_{[l]}\right\|^{2}\right),
\end{aligned}
$$

and also note that the first term $\leq e^{\|g\|^{2}}\|g\|_{\infty}^{2} h^{2}$. Next,

$$
\left\langle N_{4}[l] e\left[g_{[l]}\right), \Lambda[l] e\left[g_{[l]}\right)\right\rangle=h^{-1} \int_{\overline{l-1} h}^{l h} \bar{g}(s) d s\left\langle\int_{\overline{l-1} h}^{l h} \Gamma\left(R_{\tau}\right) d A^{\dagger}(\tau) e\left(R_{s} g_{[l]}\right), \Lambda[l] e\left(g_{[l]}\right)\right\rangle .
$$

Using the relation that for $\Delta, \Delta^{\prime} \subseteq \mathbb{R}_{+}$and $f \in L^{2}\left(\mathbb{R}_{+}\right)$,

$$
A(\Delta) \Lambda\left(\mathcal{X}_{\Delta^{\prime}}\right) e(f)=\left[\int_{\Delta \cap \Delta^{\prime}} f(\tau) d \tau+\int_{\Delta} f(\tau) d \tau \Lambda\left(\mathcal{X}_{\Delta^{\prime}}\right)\right] e(f),
$$

we have that

$$
\begin{aligned}
\left\langle\int_{\overline{l-1} h}^{l h} \Gamma\left(R_{\tau}\right) d A^{+}(\tau) e\left(R_{s} g_{[l]}\right), \Lambda[l] e\left(g_{[l]}\right)\right\rangle & =\int_{\overline{l-1} h}^{l h}\left\langle e\left(R_{\tau} R_{s} g_{[l]}\right), e\left(g_{[l]}\right)\right\rangle g(\tau) d \tau \\
+\int_{\overline{l-1} h}^{l h} g(\tau) d \tau & \left(\int_{\overline{l-1} h}^{l h} \overline{R_{\tau} R_{s} g(t)} g(t) d t\right)\left\langle e\left(R_{\tau} R_{s} g_{[l]}\right), e\left(g_{[l]}\right)\right\rangle
\end{aligned}
$$

and therefore (12) is equal to

$$
h^{-1} \int_{\overline{l-1} h}^{l h} \bar{g}(s) d s \int_{\overline{l-1} h}^{l h} g(\tau) d \tau\left\{1+\int_{\overline{l-1} h}^{l h} \overline{R_{\tau} R_{s} g(t)} g(t) d t\right\} \cdot e^{\left\langle R_{\tau} R_{s} g_{[l]}, g_{[l]}\right\rangle} .
$$

Finally we note that

$$
\begin{aligned}
& \overline{\left\langle N_{4}[l] e\left(g_{[l]}\right), \Lambda(l) e\left(g_{[l]}\right)\right\rangle} \\
& =h^{-1} \int_{\overline{l-1} h}^{l h} g(s) d s \int_{\overline{l-1} h}^{l h} \overline{g(\tau)} d \tau\left\{1+\int_{\overline{l-1} h}^{l h} R_{\tau} R_{s} g(t) \overline{g(t)} d t\right\} \cdot e^{\left\langle\overline{R_{\tau} R_{s} g_{[l]}, g_{[l]}}\right\rangle} \\
& =\left\langle N_{4}[l] e\left(g_{[l]}\right), \Lambda(l) e\left(g_{[l]}\right)\right\rangle,
\end{aligned}
$$

since $R_{\tau}^{*}=R_{\tau}$. Putting all this together in (11) we observe that

$$
\begin{aligned}
& \left\|\left[N_{4}[l]-\Lambda(l)\right] e\left(g_{[l]}\right)\right\|^{2}=\frac{1}{h} \int_{\overline{l-1} h}^{l h} \bar{g}(s) d s \int_{\overline{l-1} h}^{l h} g(\tau) d \tau e^{\left\langle R_{\tau} R_{s} g_{[l]}, g_{[l]}\right\rangle} \\
& +\int_{\overline{l-1} h}^{l h}|g(s)|^{2} d s e^{\left\|g_{[l]}\right\|^{2}}-\frac{2}{h} \int_{\overline{l-1} h}^{l h} \bar{g}(s) d s \int_{\overline{l-1} h}^{l h} g(\tau) d \tau e^{\left\langle R_{\tau} R_{s} g_{[l]}, g_{[l]}\right\rangle}+O\left(h^{2}\right) \\
\leq & \int_{\overline{l-1} h}^{l h}|g(s)|^{2} d s \exp \left(\left\|g_{[l]}\right\|^{2}\right)-\frac{1}{h} \int_{\overline{l-1} h}^{l h} \bar{g}(s) d s \int_{\overline{l-1} h}^{l h} g(\tau) d \tau e^{\left\langle R_{\tau} R_{s} g_{[l]}, g_{[l]}\right\rangle}+O\left(h^{2}\right) \\
= & \left(\frac{1}{h} \int_{\overline{l-1} h}^{l h} \bar{g}(s) d s \int_{\overline{l-1} h}^{l h}[g(s)-g(\tau)] d \tau\right) e^{\left\|g_{[l]}\right\|^{2}} \\
& +\frac{1}{h} \int_{\overline{l-1} h}^{l h} \bar{g}(s) d s \int_{\overline{l-1} h}^{l h} g(\tau) d \tau\left(e^{\left\|g_{[l]}\right\|^{2}}-e^{\left\langle R_{\tau} R_{s} g_{[l]}, g_{[l]}\right\rangle}\right)+O\left(h^{2}\right) .
\end{aligned}
$$


The first term in (13) in absolute value does not exceed

$$
\|g\|_{\infty}\left\|g^{\prime}\right\|_{\infty} \frac{e^{\|g\|^{2}}}{h} \int_{\overline{l-1} h}^{l h} d s \int_{\overline{l-1} h}^{l h}|s-\tau| d \tau=\frac{1}{6} \exp \left(\|g\|^{2}\right)\|g\|_{\infty}\left\|g^{\prime}\right\|_{\infty} h^{2},
$$

while the second term in (13) in absolute value does not exceed

$$
\begin{array}{r}
\frac{1}{h} \int_{\overline{l-1} h}^{l h}|g(s)| d s \int_{\overline{l-1} h}^{l h}|g(\tau)| d \tau\left|\exp \left(\int_{\overline{l-1} h}^{l h}|g|^{2}\right)-\exp \left\{\int_{\overline{l-1} h}^{s \wedge \tau}|g|^{2}-\int_{s \wedge \tau}^{s \vee \tau}|g|^{2}+\int_{s \vee \tau}^{l h}|g|^{2}\right\}\right| \\
\leq \frac{1}{h} \exp \left(\|g\|^{2}\right) \int_{\overline{l-1} h}^{l h}|g(s)| d s \int_{\overline{l-1} h}^{l h}|g(\tau)| d \tau\left|1-e^{-2 \int_{s \wedge \tau}^{s \vee \tau}|g|^{2}}\right| \leq 2\|g\|_{\infty}^{4} \exp \left(\|g\|^{2}\right) h^{2} .
\end{array}
$$

Combining all these together in (13), we get finally,

$$
\left\|\left(N_{4}[l]-\Lambda[l]\right) e\left(g_{[l]}\right)\right\| \leq C_{2} h .
$$

REMARK 3.2. From Lemma 3.1 (ii), it is clear that for $k=1,2$;

$$
\left[\sum_{l=1}^{n} h^{\epsilon_{k}} N_{k}(\overline{l-1} h, l h)-\Lambda_{k}(0, t)\right] e(g) \rightarrow 0
$$

as $h \rightarrow 0$ for $g \in B C^{1}\left(\mathbb{R}_{+}\right)$. In order to prove a similar result for $k=3$, 4 , one has to be a bit more careful.

Corollary 3.3. For $g \in \mathcal{M}$ and $k=1,2,3,4$ with the choices of $\epsilon_{k}$ as given in Lemma 3.1,

$$
\left[\sum_{l=1}^{n} h^{\epsilon_{k}} N_{k}(\overline{l-1} h, l h)-\Lambda_{k}(0, t)\right] e(g) \rightarrow 0
$$

as $h \rightarrow 0+$.

Proof. As we have observed above the proof for $k=1,2$ is straightforward. For the rest, we note from Proposition 2.1 that if we set

$$
\begin{aligned}
X_{t, h}^{k} e(g) & \equiv\left[\sum_{l=1}^{n} h^{\epsilon_{k}} N_{k}(\overline{l-1} h, l h)-\Lambda_{k}(0, t)\right] e(g) \\
& =\sum_{l=1}^{n}\left[h^{\epsilon_{k}} N_{k}(\overline{l-1} h, l h)-\Lambda_{k}(\overline{l-1} h, l h)\right] e(g) \\
& \equiv \sum_{l=1}^{n} M_{k}(\overline{l-1} h, l h) e(g)
\end{aligned}
$$

then

$$
\begin{aligned}
\left\|X_{t, h}^{k} e(g)\right\|^{2} & =\sum_{l=1}^{n}\left\|M_{k}(\overline{l-1} h, l h) e\left(g_{[l]}\right)\right\|^{2}\left\|e\left(g-g_{[l]}\right)\right\|^{2} \\
+ & 2 R e \sum_{l=1}^{n}\left\langle X_{\overline{l-1} h, h}^{(k)} e\left(g_{\overline{l-1} h]}\right), e\left(g_{\overline{l-1} h]}\right)\right\rangle\left\langle e\left(g_{[\overline{l-1} h}\right), M_{k}(\overline{l-1} h, l h) e\left(g_{[\overline{l-1} h}\right)\right\rangle \\
& =I_{3}+I_{4} .
\end{aligned}
$$

In the above we have used the fact that each $M_{k}$ is a martingale w.r.t. the Fock filtration, 
i.e. for $s<t$

$$
\left\langle e\left(g_{s]}\right), M_{k}(s, t) e\left(g_{s]}\right)\right\rangle=0 .
$$

This is because the L.H.S. above equals

$$
\left\langle e\left(\theta(s, t)^{-1} g_{s]}\right), M_{k}(0,1) e\left(\theta(s, t)^{-1} g_{s]}\right)\right\rangle=\left\langle e(0), M_{k}(0,1), e(0)\right\rangle=0 \quad \text { for } k=2,3
$$

in an obvious fashion and for $k=4$,

$$
\left\langle e(0), M_{4}(0,1), e(0)\right\rangle=\left\langle e(0), N_{4}(0,1), e(0)\right\rangle=\left\|\int_{0}^{1} \Gamma\left(R_{s}\right) d A(s) e(0)\right\|^{2}=0,
$$

while for $k=1$

$$
\left\langle e(0), M_{1}(0,1), e(0)\right\rangle=\left\|\int_{0}^{1} \Gamma\left(R_{s}\right) d A^{\dagger}(s) e(0)\right\|^{2}-1=\left\|A^{\dagger}(0,1) e(0)\right\|^{2}-1=0 .
$$

Next we note that, by the estimates in Lemma 3.1(ii), for $k=3,4,\left\|X_{t, h}^{(k)} e(g)\right\| \leq$ constant, independent of $h$, and therefore by using Lemma 3.1(i) in $I_{4}$ in (14), we get that $\left|I_{4}\right| \rightarrow 0$ as $h \rightarrow 0$, On the other hand, since by Lemma 3.1(ii)

$$
\left\|M_{k}(\overline{l-1} h, l h) e(g)\right\|^{2} \leq \text { Constant } \cdot h^{2},
$$

we have $\left|I_{3}\right| \rightarrow 0$ as $h \rightarrow 0$.

Corollary 3.3 essentially produces the result obtained by Attal and Pautrat [A-P2006] on exponential vectors though they used an entirely different construction. This also makes clear in what sense the "random walks" approximate various quantum noises.

4. Quantum diffusion as a limit of random walk. In this section we shall address the central result of this paper, viz. that the quantum n-step random walk $J_{t}^{(h)}(x)$ with step-size $\mathrm{h}$ in (8) converges as $h \rightarrow 0, n \rightarrow \infty$ with $n h \rightarrow t$ strongly to the solution $j_{t}(x)$ of a particular quantum stochastic differential equation, provided the basic 1-step homomorphism $\alpha(h,$.$) has suitable limiting properties. Unlike in [L-P 1988] or [A-P 2006],$ the strong convergence allows one to conclude that $\left(j_{t}\right)_{t \geq 0}$ is a flow of *-homomorphisms on the algebra $\mathcal{A}$ of observables and therefore is a "quantum diffusion".

To present the results in this section, we make two basic assumptions separately so that one knows which assumption leads to exactly which result.

Assumptions.

A1: The quantum stochastic initial value problem: for $x \in \mathcal{A}, t \geq 0, u \in h, f \in L^{2}\left(\mathbb{R}_{+}\right)$,

$$
j_{t}(x) u e(f)=x u e(f)+\int_{0}^{t} \sum_{k=1}^{4} j_{s}\left(\beta_{k}(x)\right) d \Lambda_{k}(s) . u e(f)
$$

has a unique strongly continuous solution $j_{t}: \mathcal{A} \mapsto \mathcal{A} \otimes \mathcal{B}(\mathcal{H})$. Furthermore, it is assumed that for fixed $u \in \mathfrak{h}$ and $f \in \mathcal{M} \subseteq L^{2}\left(\mathbb{R}^{+}\right)$the solution satisfies the bound:

$$
\left\|j_{t}(x) u e(f)\right\| \leq C(t, f)\|x\|\|u\| \text {. }
$$

A2: Assume furthermore that the basic *-homomorphism $\alpha(h,$.$) satisfies for k=1,2,3,4$,

$$
h^{-\eta_{k}}\left\{\alpha_{k}(h, x)-b_{k} x-h^{\epsilon_{k}} \beta_{k}(x)\right\} \rightarrow 0 \quad \text { as } h \rightarrow 0
$$

uniformly with respect to $x \in \mathcal{A}$, where $\eta_{k}=1$ for $k=1,3$ and $=1 / 2$ for $k=2,4$. 
Since we are assuming in this paper that the structure maps $\left(\beta_{k}\right)$ are bounded, assumption A1 holds ([Par 1992]).

TheOrem 4.1. Assume A1 and A2. Then $J_{t}^{(h)}(x)$, as defined in (8), converges strongly to $j_{t}(x)$ for each $x \in \mathcal{A}$ and $t \geq 0$. Thus $j_{t}: \mathcal{A} \rightarrow \mathcal{A} \otimes \mathcal{B}(\mathcal{H})$ is a unital ${ }^{*}$-homomorphic flow.

Proof. (a) From A1 it follows that for $u \in \mathfrak{h}, f \in \mathcal{M}$ and $0 \leq s<t<T, x \in \mathcal{A}$

$$
\begin{aligned}
\left\|\left[j_{t}(x)-j_{s}(x)\right] u e(f)\right\|^{2} & =\left\|\sum_{k=1}^{4} \int_{s}^{t} j_{t}\left(\beta_{k}(x)\right) \Lambda_{k}(d t) u e(f)\right\|^{2} \\
& \leq C^{\prime}(T, f) \sum_{k=1}^{4} \int_{s}^{t}\left\|j_{t}\left(\beta_{k}(x)\right) u e(f)\right\|^{2} d s \\
& \leq C^{\prime \prime}(T, f)\|u\|^{2} \sum_{k=1}^{4}\left\|\beta_{k}(x)\right\|^{2}(t-s) \\
& \leq \widetilde{C}(t, f)^{2}(t-s)\|u\|^{2}\|x\|^{2} .
\end{aligned}
$$

Thus in the definition of the strong integral in the right-hand side of (15) with respect to the partition of $[0, t)$ as given in $(8)$, one has that

$$
\begin{aligned}
\left(\sum_{k=1}^{4} \int_{0}^{t} j_{s}\left(\beta_{k}(x)\right) \Lambda_{k}(d s)-\sum_{k=1}^{4} \sum_{l=1}^{n} j_{\overline{l-1} h}\left(\beta_{k}(x)\right) \Lambda_{k}[l]\right) u e(f) & \\
& =\sum_{k=1}^{4} \int_{0}^{t}\left[j_{s}\left(\beta_{k}(x)\right)-j_{s, n}\left(\beta_{k}(x)\right)\right] \Lambda_{k}(d s) u e(f),
\end{aligned}
$$

with $j_{s, n}(y)=j_{\overline{l-1} h}(y)$ for $\overline{l-1} h \leq s<l h, l=1,2, \ldots, n$.

By using the earlier estimate, for $u \in \mathfrak{h}, f \in \mathcal{M}, x \in \mathcal{A}$ we have that

$$
\begin{aligned}
\left\|\left(\sum_{k=1}^{4} \int_{0}^{t} j_{s}\left(\beta_{k}(x)\right) \Lambda_{k}(d s)-\sum_{k=1}^{4} \sum_{l=1}^{n} j_{\overline{l-1} h}\left(\beta_{k}(x)\right) \Lambda_{k}[l]\right) u e(f)\right\|^{2} \\
\leq D^{\prime}(T, f) \sum_{k=1}^{4} \int_{0}^{t}\left\|\left[j_{s}\left(\beta_{k}(x)\right)-j_{s, n}\left(\beta_{k}(x)\right)\right] u e(f)\right\|^{2}\left(1+|f(s)|^{2}\right) d s \\
\leq \widetilde{D}(T, f) \sum_{k=1}^{4} \sum_{l=1}^{n} \int_{\overline{l-1} h}^{l h} d s\left\|\left[j_{s}\left(\beta_{k}(x)\right)-j_{\overline{l-1} h}\left(\beta_{k}(x)\right)\right] u e(f)\right\|^{2} \\
\leq \widetilde{D} \widetilde{C}^{2}\|u\|^{2} \sum_{k=1}^{4}\left\|\beta_{k}(x)\right\|^{2} \sum_{l=1}^{n} h^{2} \leq D(T, f)\|u\|^{2}\|x\|^{2} h .
\end{aligned}
$$

This establishes the fact that for $u, f$ and $x$ as above,

$$
\begin{aligned}
& \left(j_{t}(x)-x-\sum_{k=1}^{4} \sum_{l=1}^{n} j_{\overline{l-1} h}\left(\beta_{k}(x)\right) \Lambda_{k}[l]\right) u e(f) \\
& \quad=\left(\sum_{k=1}^{4} \int_{0}^{t} j_{s}\left(\beta_{k}(x)\right) \Lambda_{k}(d s)-\sum_{k=1}^{4} \sum_{l=1}^{4} j_{\overline{l-1} h}\left(\beta_{k}(x)\right) \Lambda_{k}[l]\right) u e(f)
\end{aligned}
$$


converges to 0 , uniformly w.r.t. $x \in \mathcal{A}$, as $h \rightarrow 0, n \rightarrow \infty$ with $n h \rightarrow t$. From (8), it follows that

$$
\begin{aligned}
J_{t}^{(h)}(x)-x= & \sum_{l=1}^{n}\left\{J_{l h}^{(h)}(x)-J_{\overline{l-1} h}^{(h)}(x)\right\} \\
= & \sum_{k} \sum_{l=1}^{n}\left\{J_{\overline{l-1} h}^{(h)}\left(\alpha_{k}(h, x)\right) \otimes N_{k}(\overline{l-1} h, l h)-J_{\overline{l-1} h}^{(h)}(x) \otimes b_{k} N_{k}(\overline{l-1} h, l h)\right\} \\
= & \sum_{k} \sum_{l=1}^{n} J_{\overline{l-1} h}^{(h)}\left(\alpha_{k}(h, x)-b_{k} x\right) \otimes N_{k}(\overline{l-1} h, l h) \\
= & \sum_{k} \sum_{l=1}^{n} J_{\overline{l-1} h}^{(h)}\left(\alpha_{k}(h, x)-b_{k} x-h^{\epsilon_{k}} \beta_{k}(x)\right) \otimes N_{k}[l] \\
& \left.+\sum_{k} \sum_{l=1}^{n} J_{\frac{(h)}{l-1} h}^{\left(\beta_{k}\right.}(x)\right) \otimes\left\{h^{\epsilon_{k}} N_{k}[l]-\Lambda_{k}[l]\right\}+\sum_{k} \sum_{l=1}^{n} J_{\frac{(h)}{l-1} h}\left(\beta_{k}(x)\right) \otimes \Lambda_{k}[l] .
\end{aligned}
$$

Thus for $u \in \mathfrak{h}, f \in \mathcal{M}, x \in \mathcal{A}$, and using (a), we get

$$
\begin{aligned}
{\left[J_{t}^{(h)}(x)-j_{t}(x)\right] u e(f) } & \\
= & \sum_{k} \sum_{l=1}^{n}\left(J_{l-1 h}^{(h)}\left(\alpha_{k}(h, x)-b_{k} x-h^{\epsilon_{k}} \beta_{k}(x)\right) \otimes N_{k}[l]\right) u e(f) \\
& +\sum_{k} \sum_{l=1}^{n}\left(J_{\overline{l-1} h}^{(h)}\left(\beta_{k}(x)\right) \otimes\left\{h^{\epsilon_{k}} N_{k}[l]-\Lambda_{k}[l]\right\}\right) u e(f) \\
& -\left[\sum_{k} \int_{0}^{t} j_{s}\left(\beta_{k}(x)\right) \Lambda_{k}(d s)-\sum_{k} \sum_{l=1}^{n} j_{\overline{l-1} h}\left(\beta_{k}(x)\right) \Lambda_{k}[l]\right] u e(f) \\
& +\left(\sum_{k} \sum_{l=1}^{n}\left\{J_{\frac{l}{l-1} h}^{(h)}\left(\beta_{k}(x)\right)-j_{\overline{l-1} h}\left(\beta_{k}(x)\right)\right\} \otimes \Lambda_{k}[l]\right) u e(f) \\
= & I_{1}+I_{2}+I_{3}+I_{4},
\end{aligned}
$$

where we have used the notations used in the proof of Lemma 3.1.

Next we fix $u \in \mathfrak{h}, f \in \mathcal{M}, x \in \mathcal{A}$ and then using (9) and assumption A2, we can estimate $I_{1}$ in (18):

$$
\left\|I_{1}\right\| \leq\|u\| \sum_{k=1}^{4} \sum_{l=1}^{n}\left\|\alpha_{k}(h, x)-b_{k} x-h^{\epsilon_{k}} \beta_{k}(x)\right\|\left\|N_{k}[l] e(f)\right\|
$$

which goes to zero uniformly w.r.t. $x \in \mathcal{A}$, as $h \rightarrow 0$ once we observe from Lemma 3.1 that

$$
\left\|N_{1,3}[l] e\left(f_{[l]}\right)\right\| \leq C_{1}^{\prime},\left\|N_{2,4}[l] e\left(f_{[l]}\right)\right\| \leq C_{2}^{\prime} h^{\frac{1}{2}}
$$

with constants $C_{1}^{\prime}$ and $C_{1}^{\prime}$ depending on $f$ only. 
For $I_{2}$, we shall use again Proposition 2.1 as we did in the proof of Corollary 3.3. For this we set

$$
Y_{t, h}^{k} \equiv \sum_{l=1}^{n} J_{\overline{l-1} h}^{(h)}\left(\beta_{k}(x)\right) \otimes\left\{h^{\epsilon_{k}} N_{k}[l]-\Lambda_{k}[l]\right\}
$$

and observe first that by Lemma 3.1(ii)

$$
\left\|Y_{t, h}^{k} u e\left(f_{[l]}\right)\right\| \leq \text { Constant } \cdot\|x\| \text { (independent of } h \text { ) }
$$

Thus

$$
\begin{aligned}
\left\|Y_{t, h}^{k} u e(f)\right\|^{2}= & \sum_{l=1}^{n}\left\|J_{\overline{l-1} h}^{(h)}\left(\beta_{k}(x)\right) u e\left(f_{\overline{l-1} h]}\right)\right\|^{2} \\
& \cdot\left\|\left(h^{\epsilon_{k}} N_{k}[l]-\Lambda_{k}[l]\right) e\left(f_{[l]}\right)\right\|^{2}\left\|e\left(f_{[l h}\right)\right\|^{2} \\
& +2 R e \sum_{l=1}^{n}\left\langle Y_{\overline{l-1} h, h}^{k} u e\left(f_{\overline{l-1} h]}\right), u e\left(f_{\overline{l-1} h]}\right)\right\rangle \\
& \cdot\left\langle e\left(f_{[l]}\right),\left\{h^{\epsilon_{k}} N_{k}[l]-\Lambda_{k}[l]\right\} e\left(f_{[l]}\right)\right\rangle \cdot\left\|e\left(f_{[l h}\right)\right\|^{2}
\end{aligned}
$$

which, again by virtue of Lemma 3.1 (i) and (ii) and (19) goes to zero uniformly w.r.t. $x \in \mathcal{A}$ as $h \rightarrow 0$.

Thus using the observation in the part (a) of this proof and (18), we note that given an arbitrary $\epsilon>0$, we can choose $h>0$ sufficiently small so that

$$
\left\|I_{1}+I_{2}+I_{3}\right\|<\epsilon c\|x\|,
$$

where $c$ is a constant depending only on $u, f, T$ but independent of $h$ and $x$.

(c) This last part of the proof is similar to that of theorem 3.3 in [L-P 1988]. For fixed $u \in \mathfrak{h}$ and $f \in \mathcal{M}$, define

$$
T_{t}^{(h)}(x)=J_{t}^{(h)}(x) u e(f), \quad \mathcal{T}_{t}(x)=j_{t}(x) u e(f) .
$$

First of all, note that both the maps $T_{t}^{(h))}$ and $\mathcal{T}_{t}$ are well-defined bounded linear maps from $\mathcal{A}$ into $h \otimes \mathcal{H}$. Next, we get from (18) and (20) that

$$
\left\|T_{t}^{(h)}(x)-\mathcal{T}_{t}(x)\right\|^{2} \leq 2 c^{2} \epsilon^{2}\|x\|^{2}+2 C^{2} \sum_{l=1}^{n} h \sum_{k=1}^{4}\left\|T_{\overline{l-1} h}^{(h)}\left(\beta_{k}(x)\right)-\mathcal{T}_{\overline{l-1} h}\left(\beta_{k}(x)\right)\right\|^{2},
$$

where we have used the standard estimate (page 223 [Par 1992]) for $I_{3}$ and $\mathrm{C}$ is a constant which depends only on $f$. Noting that $\beta_{k}^{\prime}$ 's are all bounded, the above leads to

$$
\begin{aligned}
\left\|T_{t}^{(h)}-\mathcal{T}_{t}\right\|^{2} & \leq 2 c^{2} \epsilon^{2}+2 C^{2} \sum_{k=1}^{4}\left\|\beta_{k}\right\|^{2} \sum_{l=1}^{n} h\left\|T_{\overline{l-1} h}^{(h)}-\mathcal{T}_{\overline{l-1} h}\right\|^{2} \\
& =2 c^{2} \epsilon^{2}+D \sum_{l=1}^{n}\left\|T_{\overline{l-1} h}^{(h)}-\mathcal{T}_{\overline{l-1} h}\right\|^{2} \cdot h
\end{aligned}
$$

where we have set $D=2 C^{2} \sum_{k=1}^{4}\left\|\beta_{k}\right\|^{2}$. As in [L-P 1988], we note that since the initial values $T_{0}^{(h)}(x)=x u e(f)=\mathcal{T}_{0}(x)$, it follows that

$$
\left\|T_{t}^{(h)}-\mathcal{T}_{t}\right\|^{2} \leq(1+h D)^{n} 2 c^{2} \epsilon^{2} \leq 2 e^{t D} c^{2} \epsilon^{2}
$$


and since $\epsilon$ was arbitrary, it follows that

$$
\lim _{h \rightarrow 0}\left\|T_{t}^{(h)}-\mathcal{T}_{t}\right\|^{2} \equiv 0
$$

or equivalently

$$
\lim _{h \rightarrow 0}\left\|J_{t}^{(h)}(x) u e(f)-j_{t}(x) u e(f)\right\|=0, \text { for } u \in \mathfrak{h}, f \in \mathcal{M}, x \in \mathcal{A} .
$$

Next, since for $t>0$ fixed and for each $h>0, J_{t}^{(h)}$ is contractive, and $\left\{J_{t}^{(h)}(x) u e(f)\right\}_{h>0}$ is strongly Cauchy in $h$, for $u \in \mathfrak{h}$ and $f \in \mathcal{M}$, it easily follows by the density of the algebraic tensor product of $\mathfrak{h}$ and vectors of the type $e(f)$ (with $f \in \mathcal{M}$ ) in $\mathfrak{h} \otimes \Gamma\left(L^{2}\left(\mathbb{R}_{+}\right)\right.$) that:

(i) for every $t>0$ and $x \in \mathcal{A},\left\{J_{t}^{(h)}(x)\right\}_{h>0}$ is strongly Cauchy on $\mathfrak{h} \otimes \mathcal{H}$,

(ii) for every $t>0$ and $x \in \mathcal{A}, J_{t}^{(h)}(x)$ converges strongly on $\mathfrak{h} \otimes \mathcal{H}$ to $j_{t}(x)$,

(iii) since each $J_{t}^{(h)}$ is a ${ }^{*}$-homomorphic unital map from $\mathcal{A}$ to $\mathcal{A} \otimes \mathcal{B}(\mathcal{H}), j_{t}(.)_{t \geq 0}$ is also a *homomorphic unital map from $\mathcal{A}$ to $\mathcal{A} \otimes \mathcal{B}(\mathcal{H})$,

(iv) since furthermore by $\mathrm{A} 1, j_{t}(x)$ satisfies the quantum stochastic differential equation (15), $j_{t}: \mathcal{A} \rightarrow \mathcal{A} \otimes \mathcal{B}(\mathcal{H})$ is a ${ }^{*}$-homomorphic flow and is the limit of the quantum random walk (8).

REMARK 4.2. (a) In the case $\beta_{k}$ 's are bounded maps, as has been assumed here, the hypothesis A1 can actually be verfied directly by an iterative construction of the solution (see for example, [E 1989] or [Par 1992] on the exponential domain i.e. on vectors of the type $u e(f), u \in \mathfrak{h}, f \in L^{2}\left(\mathbb{R}_{+}\right)$.

(b) In the original papers ([E 1989], [M-S 1990] and [G-S 1999]), the homomorphism property was also deduced by using a kind of iterative procedure, depending heavily on the boundedness of all the "structure maps" $\beta_{k}$ 's. Here we have separated the two issues, viz, the existence and the property of homomorphism. This is more natural because for various unbounded $\beta_{k}$ 's, the existence problem may be handled by using various theories of semigroups and of evolutions, whereas the scheme of approximation of the solution by a sequence of homomorphisms (called "random walks"), if it can be adapted for unbounded $\beta_{k}$ 's, will prove the homomorphism property of the solution. However, it should be emphasised that the step (c) in the proof of the theorem 4.1 has to be modified appropriately for this to succeed. This will be addressed elsewhere.

(c) From (2), (8) and (16) we find that the map $\alpha(h,):. \mathcal{A} \rightarrow \mathcal{A} \otimes \mathcal{B}\left(\mathbb{C}^{2}\right)$ is given as:

$$
\alpha(h, x)=\left(\begin{array}{ll}
\alpha_{1}(h, x) & \alpha_{2}(h, x) \\
\alpha_{3}(h, x) & \alpha_{4}(h, x)
\end{array}\right)=\left(\begin{array}{ll}
x+h \beta_{1}(x)+O(h) & \sqrt{h} \beta_{2}(x)+O\left(h^{\frac{1}{2}}\right) \\
\sqrt{h} \beta_{3}(x)+O(h) & x+\beta_{4}(x)+O\left(h^{\frac{1}{2}}\right)
\end{array}\right)
$$

and the property of homomorphism for $\alpha(h, \cdot)$ gives:

$$
\begin{aligned}
& \alpha_{1}(h, x y)=\alpha_{1}(h, x) \alpha_{1}(h, y)+\alpha_{2}(h, x) \alpha_{3}(h, y), \\
& \alpha_{2}(h, x y)=\alpha_{1}(h, x) \alpha_{2}(h, y)+\alpha_{2}(h, x) \alpha_{4}(h, y), \\
& \alpha_{3}(h, x y)=\alpha_{3}(h, x) \alpha_{1}(h, y)+\alpha_{4}(h, x) \alpha_{3}(h, y), \\
& \alpha_{4}(h, x y)=\alpha_{3}(h, x) \alpha_{2}(h, y)+\alpha_{4}(h, x) \alpha_{4}(h, y) .
\end{aligned}
$$

Combining (23) and (24) we get, for fixed $x, y \in \mathcal{A}$,

$$
x y+h \beta_{1}(x y)+O(h)=\left(x+h \beta_{1}(x)+O(h)\right)\left(y+h \beta_{1}(y)+O(h)\right),
$$


or,

$$
h\left[\beta_{1}(x y)-\beta_{1}(x) y-x \beta_{1}(y)-\beta_{2}(x) \beta_{3}(y)\right]+O(h)=0,
$$

and similarly,

$$
\begin{aligned}
& h^{\frac{1}{2}}\left[\beta_{2}(x y)-x \beta_{2}(y)-\beta_{2}(x)\left\{y+\beta_{4}(y)\right\}\right]+O\left(h^{\frac{1}{2}}\right)=0, \\
& h^{\frac{1}{2}}\left[\beta_{3}(x y)-\beta_{3}(x) y-\left\{x+\beta_{4}(x)\right\} \beta_{3}(y)\right]+O\left(h^{\frac{1}{2}}\right)=0, \\
& {\left[\beta_{4}(x y)-\beta_{4}(x) y-x \beta_{4}(y)\right]+h \beta_{3}(x) \beta_{2}(y)+O(h)=0-\beta_{4}(x) \beta_{4}(y),}
\end{aligned}
$$

leading to the familiar structure relations in the limit of $h \rightarrow 0$,

$$
\begin{aligned}
& \beta_{1}(x y)=\beta_{1}(x) y+x \beta_{1}(y)+\beta_{2}(x) \beta_{3}(y), \\
& \beta_{2}(x y)=x \beta_{2}(y)+\beta_{2}(x)\left(y+\beta_{4}(y)\right), \\
& \beta_{3}(x y)=\beta_{3}(x) y+\left(x+\beta_{4}(x)\right) \beta_{3}(y), \\
& \beta_{4}(x y)=\beta_{4}(x) y+x \beta_{4}(y)+\beta_{4}(x) \beta_{4}(y) .
\end{aligned}
$$

Similarly, the *-preservation property of $\alpha(h,$.$) leads to$

$$
\beta_{1}(x)^{*}=\beta_{1}\left(x^{*}\right), \quad \beta_{2}(x)^{*}=\beta_{3}\left(x^{*}\right), \quad \beta_{4}(x)^{*}=\beta_{4}\left(x^{*}\right) .
$$

\section{References}

[A-F-L 1982] L. Accardi, A. Frigerio and J. Lewis, Quantum Stochastic Processes, Publ. Res. Inst. Math. Sci. 18, 97-133.

[A-K 2001] L. Accardi and S. V. Kozyrev, On the structure of Markov flows, Chaos, Solution, Fractals, 12, 2639-2655.

[A-P 2006] S. Attal and Y. Pautrat, From repeated to continuous quantum interactions, Ann. H. Poincaré 7, 59-104.

[E 1989] M. P. Evans, Existence of quantum diffusions, Probab. Theory Related Fields $81,473-483$.

[G-S 1999] D. Goswami and K. B. Sinha, Hilbert modules and stochastic dilation of a quantum dynamical semigroup on a von Neumann algebra, Comm. Math. Phys. 205, 377-403.

[H-P 1986] R. L. Hudson and K. R. Parthasarathy, Unification of fermion and boson stochastic calculus, Comm. Math. Phys. 104, 457-470.

[L-P 1988] J. M. Lindsay and K. R. Parthasarathy, The passage from random walk to diffusion in quantum probability. II, Sankhyā Ser. A 50, 151-170.

[L-W 2004] J. M. Lindsay and S. Wills, Quantum stochastic operator cocycles via associated semigroups, math.FA/0512398, Math. Proc. Cambridge Phil. Soc. (to appear).

[M-S 1990] A. Mohari and K. B. Sinha, Quantum stochastic flows with infinite degrees of freedom and countable state Markov processes, Sankhyā Ser. A 52, 43-57.

[Par 1988] K. R. Parthasarathy, The passage from random walk to diffusion in quantum probability. A celebration of applied probability, J. Appl. Probab. 1988, Special Vol. 25A, 151-166.

[Par 1992] K. R. Parthasarathy, An Introduction to Quantum Stochastic Calculus, Monographs in Mathematics 85, Birkhäuser Verlag, Basel. 